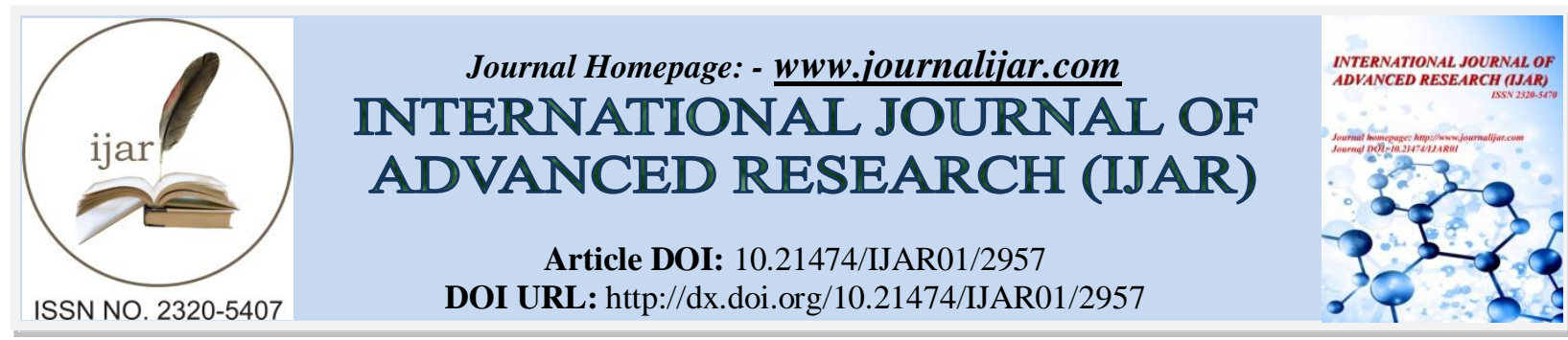

RESEARCH ARTICLE

\title{
USE OF PLANTS IN TRADITIONAL HEALTH CARE PRACTICE: AN ETHNOMEDICINAL SURVEY AT SUJANAGAR UPAZILA IN PABNA DISTRICT OF BANGLADESH.
}

Md. Anzam Ul Islam, A. H. M. Nazmul Hasan*, Afsana Awal Trina, Sk. Jahid Hasan and Md. Asaduzzaman. Department of Pharmacy, University of Asia Pacific, 74/A, Green Road, Dhaka-1215, Bangladesh.

\section{Manuscript Info}

Manuscript History

Received: 29 November 2016

Final Accepted: 26 December 2016

Published: January 2017

Key words:-

Kavirajes, Pabna, Sujanagar, Liliaceae,

Solanaceae.

\begin{abstract}
Medicinal plants are the main component in traditional medicine system and most rural people of Bangladesh still depend on folk medicine practitioners (Kavirajes) for their primary health care needs and medicinal plants used by folk medicine practitioners actually differ from region to region. The present study was designed to record the use of medicinal plants in different health ailments by the folk medicine practitioners of Sujanagar upazila(subdistrict) of Pabna district of Bangladesh. A structured survey questionnaire was prepared to obtain all the information, informed consent was obtained from the interviewer and the interview was taken in local language (Bangla) so that kavirajes could easily understand and provide necessary information. In our present study, we recorded total 63 plant species from 45 different families used by folk medicine practitioners of Sujanagar upazila in different illness. Liliaceae and Solanaceae appears the most prominent family in this study each having 3 plant species followed by Fabaceae and Acanthaceae having 2 species. Folk medicine practitioners use different parts of the plants including whole plant, leaves, fruits, barks, and rhizome. In this study, we found that leaves along with other parts of the plant had highest percentage of use (36.507\%, 23 species) and only leaf holds the second position in its usage percentage $(17.46 \%, 11$ species). This study revealed that kavirajes of Sujanagar upazila preferred oral route $(60.32 \%)$ of administration for their different medicinal formulations. There were also some formulations for topical application $(11.11 \%)$ and found some plant based formulations were also for both oral and topical application (28.57\%). This study represents many medicinal plants used by folk medicine practitioners of study area could be important source to isolate new bioactive compounds and novel drugs through extensive research in future.
\end{abstract}

Copy Right, IJAR, 2016,. All rights reserved.

\section{Introduction:-}

Human being experienced to diseases and ailments frequently during their whole life span and the nature blessed them to provide remedy to utilize plants and such type plants which are extensively used to treat health ailments are called medicinal plants. Medicinal plants are the best natural resources of traditional medicine system (Tumapa et al., 2014). Still in this modern world, $80 \%$ of people in developing countries extremely relies on traditional system 
of medicine as medicinal plants are easily accessible and cheap (Dey et al., 2014). Today plant based traditional knowledge is a recognized tool in search for new source of drug (Sahu et al., 2014). Ethnomedicinal survey which deals with ethnobotany and traditional knowledge of uses of plants is the most effective method identifying new medicinal plant and bioactive compounds, thus further study on these plants can easily lead to discovery of new therapeutic entity.

Bangladesh has a rich history of practicing traditional medicine among which unani, ayurvedic and folk medicine are quite popular in this country. Folk medicine practitioners commonly known as Kavirajes are primary health care provider to significant rural areas of the country (Salahuddin et al., 2015, Rahmatullah et al., 2010). Such traditional knowledge of uses of plants in different health ailments can be useful for planning of effective use of natural resources and conservation of biodiversity and cultural knowledge. Therefore, the main objective of this study was to document the use of plants in traditional health care practice at Sujanagar upazila (Subdistrict) in Pabna district of Bangladesh.

\section{Methods:-}

\section{Study area:-}

This study was conducted at Sujanagar upazila (subdistrict) of Pabna district. Pabna is a district having an area of 2372.5 square kilometers falls within Rajshahi division of Bangladesh. The district is roughly located between $88^{\circ} 55^{\prime}-89^{\circ} 42^{\prime} \mathrm{E}$ and $23^{\circ} 48^{\prime}-24^{\circ} 22^{\prime} \mathrm{N}$ and Sujanagar sub-district (Upazila) with an area of 334.4 square kilometers is a part of Pabna district and is located between about $89^{\circ} 23^{\prime}-89^{\circ} 38^{\prime} \mathrm{E}$ and $23^{\circ} 48^{\prime}-24^{\circ} 00^{\prime} \mathrm{N}$. The main occupation is agriculture and the major crops are paddy, jute, wheat, sugarcane, oil seeds, onion, garlic, betel leaf and pulses. The climate of Pabna is moderate and yearly temperature falls within a minimum of $9.6^{\circ} \mathrm{C}$ to a maximum of $33.9^{\circ} \mathrm{C}$; the average rainfall is $1872 \mathrm{~mm}$ (District Statistics: Pabna District, 2011).

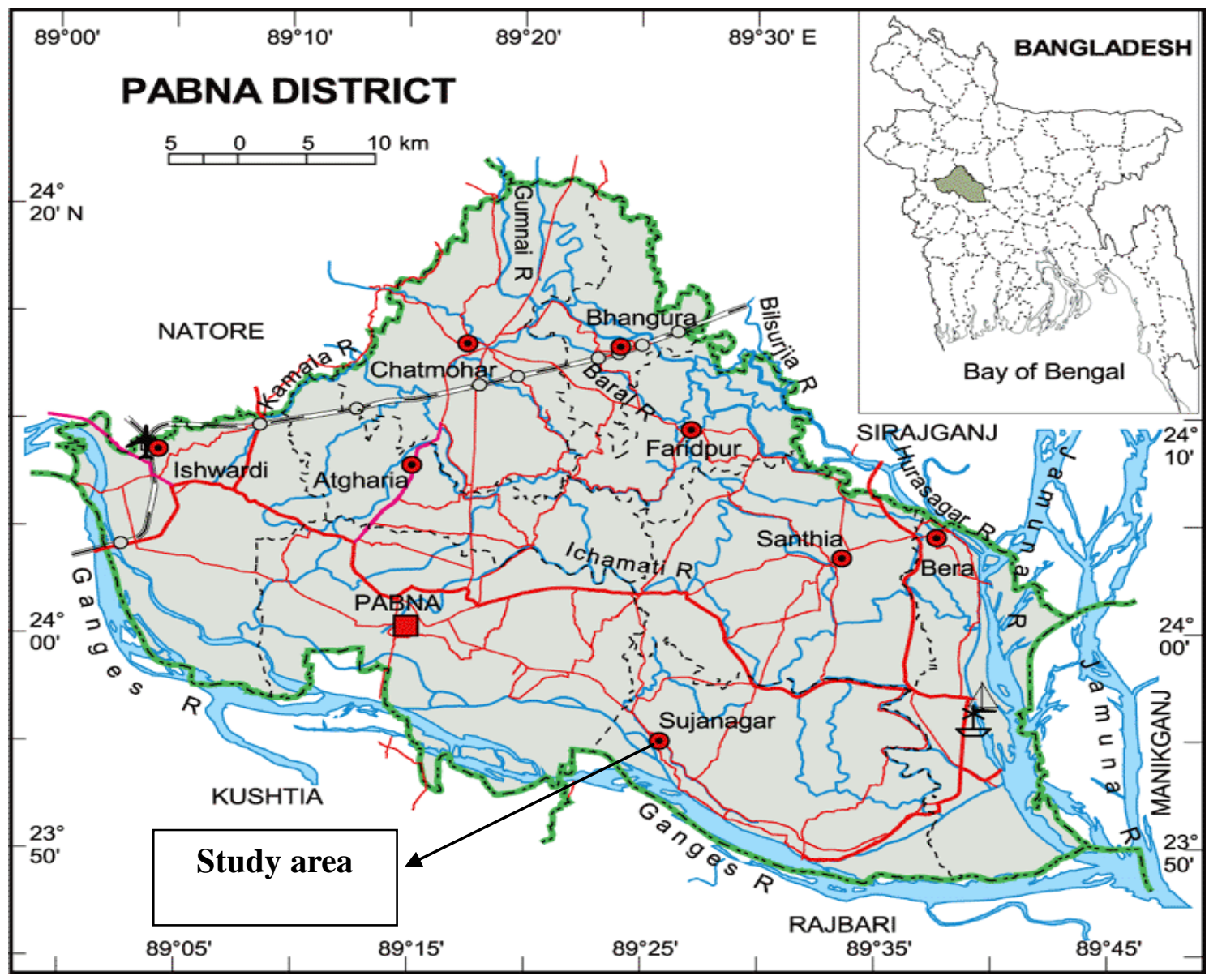

Figure 1:- Location map of Pabna district showing the study area. 


\section{Data Collection:-}

A questionnaire was prepared to collect the required data on various aspects of the study. The questions were arranged systematically and presented clearly to be easily understood by the respondents. Data on age, sex, level of education, human diseases treated, local names of plants used, parts used, methods of remedy preparations, routes of administration, noticeable adverse effects of remedies, use of antidotes for adverse effects, indigenous knowledge transfer and other uses of the ethnomedicinal plant species were gathered during the interviews. The survey was conducted in Bangla language, which was spoken both by the folk medicine practitioners (Kavirajes) as well as the surveyor and is the national language of Bangladesh. Prior to the study, informed consents were obtained from the Kavirajes that the information may be disseminated both nationally and internationally and all the information obtained was cross checked. Total 15 kavirajes were participated in this study and data were collected from $17^{\text {th }}$ August, 2016 to $24^{\text {th }}$ August, 2016.

\section{Data analysis:-}

Responses of the completed questionnaires were numerically coded and analyzed. Microsoft Office Excel 2007 program was used to process all collected information. Descriptive statistics such as frequency and percentage distribution were used to analyze data. In addition, graphs and tables were used to interpret the findings.

\section{Results:-}

\section{Distribution of plants into families:-}

In this study, total 63 plant species including herb, shrub, and tree distributed into 45 different families were recorded that is used by folk medicine practitioners (Kavirajes) of Sujanagar upazila (subdistrict) of Pabna district of Bangladesh. Liliaceae and Solanaceae appears the most prominent family in this study, each having 3 plant species followed by Fabaceae and Acanthaceae having 2 species.

Plant species and families recorded in this study are illustrated in following table along with their usage parts and dispensing procedure to people.

Table 1:- Scientific name, local name, family, parts used and indications.

\begin{tabular}{|c|c|c|c|c|c|c|}
\hline Scientific Name & Local name & Family & Part used & $\begin{array}{l}\text { Consumption/dis } \\
\text { pensing } \\
\text { procedure }\end{array}$ & Route & Use \\
\hline Abroma augusta & Ulotkombol & Sterculiaceae & Stem & $\begin{array}{l}\text { Crushed stems are } \\
\text { taken with sugar }\end{array}$ & Oral & $\begin{array}{l}\text { Debility, Infertility } \\
\text { in women due to } \\
\text { problems in uterus }\end{array}$ \\
\hline $\begin{array}{l}\text { Achyranthes } \\
\text { paniculata }\end{array}$ & Apang & Amaranthaceae & $\begin{array}{l}\text { Root, Bark, } \\
\text { Leaf }\end{array}$ & $\begin{array}{l}\text { Paste of root bark } \\
\text { and juice of roots } \\
\text { are used, } \\
\text { paste of leaves are } \\
\text { applied to the } \\
\text { affected area }\end{array}$ & $\begin{array}{l}\text { Oral, } \\
\text { Topical }\end{array}$ & $\begin{array}{l}\text { Abortion, Eczema, } \\
\text { Wound }\end{array}$ \\
\hline Aegle marmelos & Bel & Rutaceace & Leaf, Fruit & $\begin{array}{l}\text { Juice of young } \\
\text { leaves, decoction } \\
\text { of immature fruits } \\
\text { and ripe fruits are } \\
\text { taken. }\end{array}$ & Oral & $\begin{array}{l}\text { Fever, Abscess, } \\
\text { Indigestion, } \\
\text { Dysentery }\end{array}$ \\
\hline Allium сера & Piaj & Liliaceae & Bulb & $\begin{array}{l}\text { Juice of } \\
\text { bulb/scales is used }\end{array}$ & $\begin{array}{l}\text { Oral, } \\
\text { Topical }\end{array}$ & Cough, Headache \\
\hline Allium sativum & Roshun & Liliaceae & Bulb & $\begin{array}{l}\text { Juice obtained } \\
\text { from crushed bulb } \\
\text { is taken orally. }\end{array}$ & Oral & $\begin{array}{lr}\text { Pain in the chest } \\
\text { area- } & \text { usually } \\
\text { external } & \text { muscle } \\
\text { pain, } & \text { Piles, } \\
\text { Rheumatism }\end{array}$ \\
\hline $\begin{array}{l}\text { Aloe } \\
\text { barbadensis Mill }\end{array}$ & Ghritokumari & Asphodelaceae & Leaf & $\begin{array}{l}\text { Juice of leaves is } \\
\text { applied to the } \\
\text { affected area } \\
\text { and leaf mucilage }\end{array}$ & Topical & $\begin{array}{l}\text { Sexually } \\
\text { transmitted } \\
\text { diseases in men, } \\
\text { Skin disorders, }\end{array}$ \\
\hline
\end{tabular}




\begin{tabular}{|c|c|c|c|c|c|c|}
\hline & & & & is used & & $\begin{array}{lr}\text { Piles, } & \text { Menstrual } \\
\text { disease, } & \text { Sexual } \\
\text { problems } & \\
\end{array}$ \\
\hline Ananus comosus & Anarosh & Bromeliaceae & Fruit, Leaf & $\begin{array}{l}\text { Juice of unripe } \\
\text { fruit, ripe fruit and } \\
\text { leaves is used }\end{array}$ & Oral & $\begin{array}{l}\text { Abortion, Cough, } \\
\text { Diuretic, Fever, } \\
\text { Helminthiasis. }\end{array}$ \\
\hline $\begin{array}{l}\text { Andrographis } \\
\text { paniculata Nees. }\end{array}$ & Kalomegh & Acanthaceae & $\begin{array}{l}\text { Leaf, Stem, } \\
\text { Root }\end{array}$ & $\begin{array}{l}\text { Root paste is taken } \\
\text { with sugar for liver } \\
\text { disorders. Leaf } \\
\text { paste mixed with } \\
\text { sugar is taken for } \\
\text { helminthiasis. } \\
\text { Stem is taken with } \\
\text { sugar to reduce } \\
\text { acidity. }\end{array}$ & Oral & $\begin{array}{l}\text { Liver disorders, } \\
\text { Helminthiasis, } \\
\text { Gastric acidity }\end{array}$ \\
\hline $\begin{array}{l}\text { Aristolochia } \\
\text { indica L. }\end{array}$ & Ishwarmul & Aristolochiaceae & Root & $\begin{array}{l}\text { Crushed roots are } \\
\text { used }\end{array}$ & Topical & Snake bite \\
\hline $\begin{array}{l}\text { Artocarpus } \\
\text { heterophyllus } \\
\text { Lamk. }\end{array}$ & Kathal & Moraceae & $\begin{array}{l}\text { Bark, Leaf, } \\
\text { Root }\end{array}$ & $\begin{array}{l}\text { Juice made from } \\
\text { young leaves } \\
\text { young roots and } \\
\text { juice made from } \\
\text { bark is also taken }\end{array}$ & Oral & $\begin{array}{l}\text { Asthma, Itches } \\
\text { Diarrhea, } \\
\text { Excessive } \\
\text { menstrual } \\
\text { discharge }\end{array}$ \\
\hline $\begin{array}{l}\text { Asparagus } \\
\text { racemosus }\end{array}$ & Satamuli & Liliaceae & $\begin{array}{l}\text { Whole plant, } \\
\text { roots }\end{array}$ & $\begin{array}{l}\text { An amulet } \\
\text { containing whole } \\
\text { plant is tied around } \\
\text { the waist, } \\
\text { Root paste mixed } \\
\text { with sugar is taken } \\
\text { for constipation }\end{array}$ & $\begin{array}{l}\text { Oral, } \\
\text { Topical }\end{array}$ & $\begin{array}{l}\text { Constipation, } \\
\text { Impotency in men. }\end{array}$ \\
\hline $\begin{array}{l}\text { Averrhoa } \\
\text { carambola }\end{array}$ & Kamranga & Oxalidaceae & Fruits & Fruits are eaten & Oral & $\begin{array}{l}\text { Jaundice, Fever, } \\
\text { Piles }\end{array}$ \\
\hline $\begin{array}{l}\text { Azadirachta } \\
\text { indica }\end{array}$ & Neem & Meliaceae & Leaf, Bark & $\begin{array}{l}\text { Paste of the leaves } \\
\text { is applied to the } \\
\text { affected area and } \\
\text { powder } \\
\text { of bark is taken. }\end{array}$ & $\begin{array}{l}\text { Oral, } \\
\text { Topical }\end{array}$ & $\begin{array}{l}\text { Rheumatic fever, } \\
\text { Itches, Ring worm, } \\
\text { Helminthiasis, } \\
\text { Chicken pox. }\end{array}$ \\
\hline $\begin{array}{l}\text { Blumea } \\
\text { membranacea } \\
\text { DC. }\end{array}$ & Ukra, Uhra & Asteraceae & Leaf, stem & $\begin{array}{l}\text { Juice from stem } \\
\text { and leaves are } \\
\text { applied to the scalp }\end{array}$ & Topical & Fever with mucus \\
\hline $\begin{array}{l}\text { Cajanus } \\
\text { cajan }(\text { L).Huth }\end{array}$ & Aral & Fabaceae & Leaf & $\begin{array}{l}\text { Young leaf juice is } \\
\text { taken. }\end{array}$ & Oral & Jaundice, Diabetes \\
\hline $\begin{array}{l}\text { Calotropis } \\
\text { gigantea }\end{array}$ & Akondo & Apocynaceae & $\begin{array}{l}\text { Root, Leaf, } \\
\text { Bark }\end{array}$ & $\begin{array}{l}\text { Leaves are taken } \\
\text { during pneumonia. } \\
\text { Leaves are warmed } \\
\text { in oil and applied } \\
\text { to painful areas }\end{array}$ & $\begin{array}{l}\text { Oral , } \\
\text { Topical }\end{array}$ & $\begin{array}{l}\text { Ulcer, Tooth pain, } \\
\text { Chronic dysentery, } \\
\text { Cold, Asthma, Pain }\end{array}$ \\
\hline $\begin{array}{l}\text { Carica papaya } \\
\text { L. }\end{array}$ & Pepe & Caricaceae & Fruit, Latex & $\begin{array}{l}\text { Fruit juice ,Ripe } \\
\text { fruits are taken and } \\
\text { latex is used }\end{array}$ & $\begin{array}{l}\text { Oral, } \\
\text { Topical }\end{array}$ & $\begin{array}{l}\text { Constipation, } \\
\text { Itches, Indigestion, } \\
\text { Liver disease, } \\
\text { Diarrhea }\end{array}$ \\
\hline $\begin{array}{l}\text { Cassia sophera } \\
\text { L. }\end{array}$ & Kolkashundia & Fabaceae & Whole plants & $\begin{array}{l}\text { Paste of whole } \\
\text { plant is applied to } \\
\text { affected area }\end{array}$ & Topical & Eczema. \\
\hline Centella asiatica & $\begin{array}{l}\text { Chaka } \\
\text { thankuni }\end{array}$ & Apiaceae & Leaf, Stem & $\begin{array}{l}\text { Juice obtained } \\
\text { from } \\
\text { leaves and stems is }\end{array}$ & Oral & Stomach disorders \\
\hline
\end{tabular}




\begin{tabular}{|c|c|c|c|c|c|c|}
\hline & & & & used. & & \\
\hline $\begin{array}{l}\text { Cissus } \\
\text { quadrangularis } \\
\text { L. }\end{array}$ & Harjora & Vitaceae & Leaf, Stem & $\begin{array}{l}\text { Paste of leaf and } \\
\text { stem is applied to } \\
\text { fractures }\end{array}$ & Topical & Bone fracture \\
\hline $\begin{array}{l}\text { Coccinia } \\
\text { grandis } \\
\text { Voigt }\end{array}$ & Telakucha & Cucurbitaceae & Leaf & $\begin{array}{l}\text { Vegetables made } \\
\text { from young leaves } \\
\text { are eaten }\end{array}$ & Oral & Diabetes, Fever \\
\hline $\begin{array}{l}\text { Curcuma longa } \\
\text { L. }\end{array}$ & Holud & Zingiberaceae & Rhizome & $\begin{array}{l}\text { Raw rhizome is } \\
\text { taken orally, paste } \\
\text { of rhizome is } \\
\text { applied to the } \\
\text { affected area }\end{array}$ & $\begin{array}{l}\text { Oral, } \\
\text { topical }\end{array}$ & $\begin{array}{l}\text { Wet dream, } \\
\text { Scabies, Eczema }\end{array}$ \\
\hline Cuscuta reflexa & Aloklata & Convolvulaceae & Leaf & $\begin{array}{l}\text { Juice of the leaves } \\
\text { is taken }\end{array}$ & Oral & Dysentery \\
\hline $\begin{array}{l}\text { Cynodon } \\
\text { dactylon }\end{array}$ & Durva Ghash & Poaceae & Whole plant & $\begin{array}{l}\text { Juice of the plant is } \\
\text { taken with milk } \\
\text { and applied to } \\
\text { wound }\end{array}$ & $\begin{array}{l}\text { Oral, } \\
\text { Topical }\end{array}$ & Wounds, Acne. \\
\hline Datura metel L & Kalodhutura & Solanaceae & $\begin{array}{l}\text { Leaf, Juice } \\
\text { from } \\
\text { stalk of fruits }\end{array}$ & $\begin{array}{l}\text { Macerated leaves } \\
\text { are taken with } \\
\text { juice from stalk of } \\
\text { fruits }\end{array}$ & Oral & $\begin{array}{l}\text { Antidote } \\
\text { poisoning }\end{array}$ \\
\hline Eclipta prostrata & Kalokeshi & Asteraceae & $\begin{array}{l}\text { Leaf, Fruit, } \\
\text { Flower }\end{array}$ & $\begin{array}{l}\text { Paste made from } \\
\text { leaves is used in } \\
\text { affected area. } \\
\text { Juice made from } \\
\text { whole plants are } \\
\text { used }\end{array}$ & Oral & $\begin{array}{l}\text { Jaundice, Asthma, } \\
\text { Gallbladder stone, } \\
\text { Wounds, Itches, } \\
\text { Skin diseases, } \\
\text { Constipation. }\end{array}$ \\
\hline $\begin{array}{l}\text { Ficus racemosa } \\
\text { L. }\end{array}$ & $\begin{array}{l}\text { Dumur, } \\
\text { Joggodumur }\end{array}$ & Moraceae & Fruits & $\begin{array}{l}\text { Paste of fruit is } \\
\text { taken with milk }\end{array}$ & Oral & Diabetes \\
\hline $\begin{array}{l}\text { Garcinia cowa } \\
\text { Raxb. }\end{array}$ & Kaufol & Clusiaceae & Fruits & Fruits are eaten & Oral & Cold, Coughs \\
\hline $\begin{array}{l}\text { Heliotropium } \\
\text { indicum }\end{array}$ & Hatishur & Boraginaceae & Root & $\begin{array}{l}\text { Paste of root is } \\
\text { mixed with sugar } \\
\text { or honey }\end{array}$ & Oral & $\begin{array}{l}\text { Wet dreams, STDs, } \\
\text { Swelling of knees, } \\
\text { Joint pain }\end{array}$ \\
\hline $\begin{array}{l}\text { Hibiscus } \\
\text { rosasinensis }\end{array}$ & Joba & Malvaceae & Flower, Leaf & $\begin{array}{l}\text { Paste of flower is } \\
\text { applied on fresh } \\
\text { cut and extract of } \\
\text { flower is taken }\end{array}$ & $\begin{array}{l}\text { Oral, } \\
\text { Topical }\end{array}$ & $\begin{array}{l}\text { Wound, Irregular } \\
\text { menstruation. }\end{array}$ \\
\hline $\begin{array}{l}\text { Ipomoea } \\
\text { mauritiana Jacq. }\end{array}$ & Bhuikumra & Convolvulaceae & Root & $\begin{array}{ll}\text { Root paste } & \text { is } \\
\text { applied to hair } & \& \\
\text { penis } & \end{array}$ & Topical & $\begin{array}{l}\text { Blackening of hair } \\
\text { in men and women, } \\
\text { Erectile } \\
\text { dysfunction }\end{array}$ \\
\hline $\begin{array}{l}\text { Justicia } \\
\text { adhatoda L. }\end{array}$ & Basok & Acanthaceae & Leaf & $\begin{array}{l}\text { Tablets made from } \\
\text { paste are taken }\end{array}$ & Oral & Whooping cough \\
\hline $\begin{array}{l}\text { Kalanchoe } \\
\text { pinnata (Lam.) } \\
\text { Pers. }\end{array}$ & Patharkuchi & Crassulaceae & Leaf & $\begin{array}{l}\text { Leaves are chewed } \\
\text { with salt to } \\
\text { dissolve gall } \\
\text { bladder stones, } \\
\text { Leaf paste is } \\
\text { applied topically. }\end{array}$ & $\begin{array}{l}\text { Oral, } \\
\text { Topical }\end{array}$ & $\begin{array}{l}\text { Remove } \\
\text { gallbladder stones, } \\
\text { Stomach pain, } \\
\text { Headache }\end{array}$ \\
\hline $\begin{array}{l}\text { Lawsonia } \\
\text { inermis L. }\end{array}$ & Mehedi & Lythraceae & Leaf & $\begin{array}{l}\text { Juice from leaves } \\
\text { is taken and paste } \\
\text { from leaves are } \\
\text { applied to } \\
\text { the affected area }\end{array}$ & $\begin{array}{l}\text { Oral, } \\
\text { Topical }\end{array}$ & $\begin{array}{lr}\text { Cuts and } & \text { wounds, } \\
\text { Cracked } & \text { skin, } \\
\text { Diabetes, } & \text { Burning } \\
\text { sensations during } \\
\text { urination. }\end{array}$ \\
\hline Leucas aspera & Dondo kolosh & Lamiaceae & Stem & Crushed stems is & Oral & Diarrhea, \\
\hline
\end{tabular}




\begin{tabular}{|c|c|c|c|c|c|c|}
\hline & & & & $\begin{array}{l}\text { taken usually with } \\
\text { parts of other } \\
\text { medicinal } \\
\text { Plants } \\
\text { (Azadirachta } \\
\text { indica, Coccinia } \\
\text { cordifolia) }\end{array}$ & & $\begin{array}{l}\text { purifier, Loss of } \\
\text { appetite, } \\
\text { Indigestion, Skin } \\
\text { disease }\end{array}$ \\
\hline $\begin{array}{l}\text { Mangifera } \\
\text { indica }\end{array}$ & Aam & Anacardiaceae & $\begin{array}{l}\text { Young } \\
\text { leaves, } \\
\text { Buds }\end{array}$ & $\begin{array}{l}\text { Sap of young } \\
\text { leaves is applied to } \\
\text { the eyes, a syrup } \\
\text { made } \\
\text { from the buds } \\
\text { along with Musa } \\
\text { sapientum and } \\
\text { ghee is taken } \\
\text { orally }\end{array}$ & $\begin{array}{l}\text { Oral, } \\
\text { Topical }\end{array}$ & $\begin{array}{l}\text { Conjunctivitis, } \\
\text { Passing of sperm } \\
\text { with urine. }\end{array}$ \\
\hline Mentha viridis & Pudina & Lebiatae & Whole plant & $\begin{array}{l}\text { Juice of the plants } \\
\text { is taken }\end{array}$ & Oral & $\begin{array}{l}\text { Metabolic disorder, } \\
\text { Gastritis }\end{array}$ \\
\hline $\begin{array}{l}\text { Mimosa pudica } \\
\text { L. }\end{array}$ & Lojjaboti & Mimosaceae & $\begin{array}{l}\text { Leaf, Root, } \\
\text { Fruit }\end{array}$ & $\begin{array}{l}\text { Leaf juice are used } \\
\text { externally and root } \\
\text { and fruit juices are } \\
\text { taken with honey }\end{array}$ & $\begin{array}{l}\text { Oral, } \\
\text { Topical }\end{array}$ & $\begin{array}{lr}\text { Piles, } & \text { Boils, } \\
\text { Dysentery, } & \text { Bone } \\
\text { injuries } & \text { as } \\
\text { painkiller, } & \text { Tumor } \\
\text { for early burst }\end{array}$ \\
\hline $\begin{array}{l}\text { Moringa oleifera } \\
\text { Lam. }\end{array}$ & Sogina & Moringaceae & $\begin{array}{l}\text { Leaf, Stem, } \\
\text { Root }\end{array}$ & $\begin{array}{l}\text { Juice obtained } \\
\text { from crushed } \\
\text { leaves and stems is } \\
\text { taken. }\end{array}$ & Oral & $\begin{array}{l}\text { Jaundice, } \\
\text { Chickenpox, } \\
\text { Paralysis, Fever }\end{array}$ \\
\hline $\begin{array}{l}\text { Mormordica } \\
\text { charantea } L .\end{array}$ & Korola & Cucurbitaceae & Leaf, Fruit & $\begin{array}{l}\text { Juice made from } \\
\text { leaves is used. } \\
\text { Curry made from } \\
\text { unripe fruit are } \\
\text { eaten }\end{array}$ & Oral & $\begin{array}{l}\text { Chickenpox, } \\
\text { Rheumatism, } \\
\text { Diabetes }\end{array}$ \\
\hline Nigella sativa & Kalojira & Ranunculaceae & Seed & $\begin{array}{l}\text { Paste of seeds or } \\
\text { seeds are taken } \\
\text { along with water or } \\
\text { honey } \\
\text { and oil obtained } \\
\text { from seeds are } \\
\text { applied externally. }\end{array}$ & $\begin{array}{l}\text { Oral, } \\
\text { Topical }\end{array}$ & $\begin{array}{l}\text { Rheumatic fever, } \\
\text { Hypertension, Skin } \\
\text { disease }\end{array}$ \\
\hline $\begin{array}{l}\text { Nyctanthes } \\
\text { arbortristis } L .\end{array}$ & Sheuli & Verbenaceae & Leaf & $\begin{array}{lr}\text { Boiled } & \text { and } \\
\text { macerated } & \text { leaves } \\
\text { are taken } & \end{array}$ & Oral & $\begin{array}{l}\text { Fever due to } \\
\text { metabolic } \\
\text { imbalances in the } \\
\text { body. }\end{array}$ \\
\hline $\begin{array}{l}\text { Ocimum } \\
\text { sanctum }\end{array}$ & Tulsi & Lamiaceae & Leaf, Root & $\begin{array}{l}\text { Juice of leaves are } \\
\text { taken with honey }\end{array}$ & Oral & $\begin{array}{l}\text { Rheumatic pain, } \\
\text { Coughs, } \\
\text { Constipation, } \\
\text { Snake bite }\end{array}$ \\
\hline $\begin{array}{l}\text { Ocimum } \\
\text { tenuiflorum L. }\end{array}$ & Kalo tulsi & Lamiaceae & Leaf & $\begin{array}{l}\text { Juice of leaves is } \\
\text { mixed with sugar } \\
\text { and eaten }\end{array}$ & Oral & $\begin{array}{l}\text { Coughs, } \\
\text { Dysentery, } \\
\text { Diuretics, Diabetes }\end{array}$ \\
\hline Paedaria foetida & $\begin{array}{l}\text { Gondho } \\
\text { vadule }\end{array}$ & Rubiaceae & Leaf & Leaf juice is taken & Oral & $\begin{array}{l}\text { Dysentery, } \\
\text { Metabolic disorder, } \\
\text { Cold, Arthritis }\end{array}$ \\
\hline $\begin{array}{l}\text { Phyllanthus } \\
\text { emblica } L .\end{array}$ & Amlaki & Euphorbiaceae & Fruit & $\begin{array}{lll}\text { Ripe } & \text { fruits } & \text { and } \\
\text { dried } & \text { fruits } & \text { are } \\
\text { taken } & & \end{array}$ & Oral & $\begin{array}{lr}\text { Fatigue, } & \text { Thirst, } \\
\text { Burning } & \text { sensation } \\
\text { during } & \text { urination, } \\
\text { Biliary } & \text { problem, }\end{array}$ \\
\hline
\end{tabular}




\begin{tabular}{|c|c|c|c|c|c|c|}
\hline & & & & & & Diabetes \\
\hline $\begin{array}{l}\text { Plumbago } \\
\text { zeylanica } L .\end{array}$ & Kalpanath & Plumbaginaceae & Leaf & $\begin{array}{l}\text { Paste of leaves is } \\
\text { taken in empty } \\
\text { stomach }\end{array}$ & Oral & $\begin{array}{l}\text { Infertility in } \\
\text { women }\end{array}$ \\
\hline $\begin{array}{l}\text { Psidium guajava } \\
\text { L. }\end{array}$ & Piyara & Myrtaceae & $\begin{array}{l}\text { Leaf, Fruit, } \\
\text { Bark }\end{array}$ & $\begin{array}{l}\text { Fruits and juice } \\
\text { from leaves and } \\
\text { stem barks } \\
\text { are taken and } \\
\text { decoction of leaves } \\
\text { is taken. }\end{array}$ & Oral & $\begin{array}{l}\text { Diarrhea, Debility, } \\
\text { Bloody dysentery, } \\
\text { Toothache, Worm }\end{array}$ \\
\hline $\begin{array}{l}\text { Punica } \\
\text { granatum }\end{array}$ & Dalim & Lythraceae & Fruit, Leaf & $\begin{array}{l}\text { Fruit and leaf juice } \\
\text { is taken }\end{array}$ & Oral & $\begin{array}{l}\text { Anemia, Erectile } \\
\text { dysfunction, } \\
\text { Osteoarthritis, } \\
\text { Dysentery }\end{array}$ \\
\hline $\begin{array}{l}\text { Rauwolfia } \\
\text { canescens } L .\end{array}$ & Boro chanda & Apocynaceae & Root & $\begin{array}{l}\text { Paste of roots is } \\
\text { applied to the } \\
\text { affected areas } \\
\text { and crushed roots } \\
\text { taken. }\end{array}$ & $\begin{array}{l}\text { Topical, } \\
\text { Oral }\end{array}$ & Snake bite \\
\hline $\begin{array}{l}\text { Rauwolfia } \\
\text { serpentina (L.) } \\
\text { Benth. ex Kurz }\end{array}$ & Chotochanda & Apocynaceae & Root & $\begin{array}{l}\text { Paste of roots is } \\
\text { applied to the } \\
\text { affected areas } \\
\text { and crushed roots } \\
\text { taken. }\end{array}$ & $\begin{array}{l}\text { Topical } \\
\text {, oral }\end{array}$ & Snake bite \\
\hline $\begin{array}{l}\text { Solanum } \\
\text { surattenseBurm. } \\
f .\end{array}$ & Choroibaegun & Solanaceae & Fruits & $\begin{array}{l}\text { Macerated fruits } \\
\text { are taken }\end{array}$ & Oral & $\begin{array}{l}\text { Tumor in humans, } \\
\text { Swelling of throat } \\
\text { in cattle. }\end{array}$ \\
\hline Syzygium cumini & Jam & Myrtaceae & Bark ,seed & $\begin{array}{l}\text { Paste made from } \\
\text { the bark is used } \\
\text { externally, } \\
\text { dry seed dust } \\
\text { mixed with normal } \\
\text { water taken }\end{array}$ & $\begin{array}{l}\text { Oral, } \\
\text { Topical }\end{array}$ & $\begin{array}{l}\text { Dysentery, Wound, } \\
\text { Diabetes }\end{array}$ \\
\hline $\begin{array}{l}\text { Syzygium } \\
\text { malaccense }\end{array}$ & Jamrul & Myrtaceae & Roots & $\begin{array}{l}\text { Macerated roots } \\
\text { are taken }\end{array}$ & Oral & Helminthiasis. \\
\hline $\begin{array}{l}\text { Terminalia } \\
\text { arjuna }\end{array}$ & Arjun & Combretaceae & Bark & $\begin{array}{l}\text { Bark boiled in } \\
\text { water is taken and } \\
\text { paste of bark is } \\
\text { applied }\end{array}$ & $\begin{array}{l}\text { Oral, } \\
\text { Topical }\end{array}$ & $\begin{array}{l}\text { Chest pain due to } \\
\text { heart disorders, } \\
\text { Burning } \\
\text { sensations during } \\
\text { urination, Bone } \\
\text { fracture. }\end{array}$ \\
\hline $\begin{array}{l}\text { Terminalia } \\
\text { bellirica } \\
\text { (Gaertn.) }\end{array}$ & Bohera & Combretaceae & Fruit, Seed & $\begin{array}{l}\text { Fruits are eaten, oil } \\
\text { extracted from the } \\
\text { seeds and used }\end{array}$ & $\begin{array}{l}\text { Oral, } \\
\text { Topical }\end{array}$ & $\begin{array}{l}\text { Loss of appetite, } \\
\text { Headache, } \\
\text { Rheumatic fever }\end{array}$ \\
\hline $\begin{array}{l}\text { Terminalia } \\
\text { chebula Retz. }\end{array}$ & Haritaki & Combretaceae & Fruit & $\begin{array}{l}\text { Ripe fruits and } \\
\text { unripe fruits are } \\
\text { used }\end{array}$ & Oral & $\begin{array}{l}\text { Constipation, } \\
\text { Indigestion } \\
\text { Rheumatism and } \\
\text { Urinary disease. }\end{array}$ \\
\hline $\begin{array}{l}\text { Tinospora } \\
\text { cordifolia }\end{array}$ & Pipolti & Menispermaceae & Stem & $\begin{array}{lll}\text { Plant } & \text { juice } & \text { and } \\
\text { stem } & \text { juice } & \text { are } \\
\text { taken } & & \\
\end{array}$ & Oral & $\begin{array}{l}\text { Swelling, Gastric } \\
\text { trouble and ulcers }\end{array}$ \\
\hline Vitex negundo & Nishinda & Lamiaceae & Root, Leaf & $\begin{array}{l}\text { Tablets made from } \\
\text { leaves are taken }\end{array}$ & Oral & $\begin{array}{l}\text { To increase } \\
\text { memory, Bloating, } \\
\text { Rheumatism, } \\
\text { Edema, } \\
\text { Helminthiasis, } \\
\text { Throat infections, } \\
\text { Piles, Loss of }\end{array}$ \\
\hline
\end{tabular}




\begin{tabular}{|c|c|c|c|c|c|c|}
\hline & & & & & & appetite, Fever \\
\hline Vitis pentagona & Sonatola & Vitaceae & Flower, Leaf & $\begin{array}{l}\text { Paste of flower is } \\
\text { taken and leaf } \\
\text { paste is taken with } \\
\text { salt }\end{array}$ & Oral & Rheumatic fever \\
\hline $\begin{array}{l}\text { Withania } \\
\text { somnifera (L.) } \\
\text { Dunal }\end{array}$ & Ashwagandha & Solanaceae & Fruits & $\begin{array}{l}\text { Mixture of fruit, } \\
\text { leaf and stem is } \\
\text { taken }\end{array}$ & Oral & Sex stimulant \\
\hline $\begin{array}{l}\text { Zingiber } \\
\text { officinale Roscoe }\end{array}$ & Ada & Zingiberaceae & Rhizome & $\begin{array}{l}\text { Juice from rhizome } \\
\text { is applied to the } \\
\text { scalp daily. }\end{array}$ & Topical & $\begin{array}{l}\text { Depression, Cough } \\
\& \text { cold }\end{array}$ \\
\hline $\begin{array}{l}\text { Ziziphus jujuba } \\
\text { Mill }\end{array}$ & Boroi & Rhamnaceae & Leaf, fruits & $\begin{array}{l}\text { Leaves and fruits } \\
\text { are taken }\end{array}$ & Oral & Hypertension \\
\hline
\end{tabular}

A report described the wide therapeutic use of different plant families throughout the world and many of these plants from different families are also used in the study area (Joy et al,. 1998).

Used plant parts:-

The various plant parts were used included whole plants, leafs, roots, barks, stems, seeds, fruits, and rhizome. The Kavirajes used different modes of preparation for using a particular plant or plant parts. Leaves along with other parts are most commonly used in different methods had highest percentage of use (36.507\%, 23 species) and only leaf holds the second position in its usage percentage $(17.46 \%, 11$ species). The following table summarizes percent of curative plant parts used in different health ailments by kavirajes.

Table 2:- Number of the medicinal plants with their curative parts and percentage.

\begin{tabular}{|c|c|c|c|}
\hline Serial No. & Curative plant parts & No. of species & Percentage (\%) \\
\hline 1. & Leaf only & 11 & 17.46 \\
\hline 2. & Fruit & 07 & 11.11 \\
\hline 3. & Seed & 01 & 1.59 \\
\hline 4. & Root & 06 & 9.52 \\
\hline 5. & Whole plant & 04 & 6.35 \\
\hline 6. & Leaf with other parts & 23 & 36.51 \\
\hline 7. & Flower & 00 & 0 \\
\hline 8. & Bark & 01 & 1.59 \\
\hline 9. & Wood & 00 & 0 \\
\hline 10. & Rhizome & 02 & 3.17 \\
\hline 11. & Stem & 03 & 3.76 \\
\hline 12. & Bulb & 02 & 1.17 \\
\hline 13. & Bark \& Seed & 01 & 1.59 \\
\hline 14. & Fruit \& Seed & 01 & 1.59 \\
\hline 15. & Fruit \& Latex & 01 & \\
\hline
\end{tabular}

Percent of plant use in different Ailments:-

In this study, we recorded 63 different plant species from different plant family which are used by folk medicine practitioners in their regular practice. We calculated the percent of different plant used in different health ailments and summarized in the following table. (Table: 3 ).

Table 3: Board categories of the ailments and percentage of plants used in each category

\begin{tabular}{|c|l|c|c|}
\hline Serial No. & Board categories of ailments & No of species & Percentage (\%) of plant used \\
\hline 1. & Gastrointestinal disorders & 33 & 52.38 \\
\hline 2. & Cold and Flu related ailments & 18 & 28.57 \\
\hline 3. & $\begin{array}{l}\text { Pain, Inflammation and Burning } \\
\text { sensation }\end{array}$ & 22 & 34.92 \\
\hline 4. & Sexual problems & 15 & 23.81 \\
\hline 5. & Skin diseases & 12 & 19.05 \\
\hline 6. & Respiratory tract disorders & 4 & 6.35 \\
\hline
\end{tabular}




\begin{tabular}{|c|l|c|c|}
\hline 7. & Cardiovascular diseases & 6 & 9.52 \\
\hline 8. & Nerve disorders & 4 & 6.35 \\
\hline 9. & Oral and larynx health diseases & 4 & 6.35 \\
\hline 10 & Hepatic problems & 8 & 12.70 \\
\hline 11. & Antiseptic purposes & 10 & 15.87 \\
\hline 12. & Wound and Blood disorders & 11 & 17.46 \\
\hline 13. & Bone related ailments & 15 & 23.81 \\
\hline 14. & Renal diseases & 3 & 4.76 \\
\hline 15. & Miscellaneous & 15 & 23.80 \\
\hline
\end{tabular}

In this study, we revealed that most plant species were used in the treatment of different gastrointestinal tract disorders (33 species, $52.38 \%$ ) followed by pain, inflammation and burning sensation (22 species, $34.92 \%$ ), cold and flu related ailments (18 species, $28.57 \%$ ). We also found that a single plant species or its different parts is being used in the treatment of many diseases. As a medicinal plant contains lots of chemical species, so a single medicinal plant or its different parts may have many therapeutic potential and that's why the total percentage of plants used in different diseases is more than 100 percent. Usages of plants to treat some diseases by kavirajes in our study area were also interesting.

\section{Percent of administration routes of plant species:-}

Folk medicine practitioners preferred oral routes of administration. In our study we found that, $60.32 \%$ (38 species) of their medicinal formulations were for oral route. Some of their formulations were for topical application $(11.11 \%$, 7 species). We also found some plant species formulations were for both oral and topical application (18 species, $28.57 \%)$.

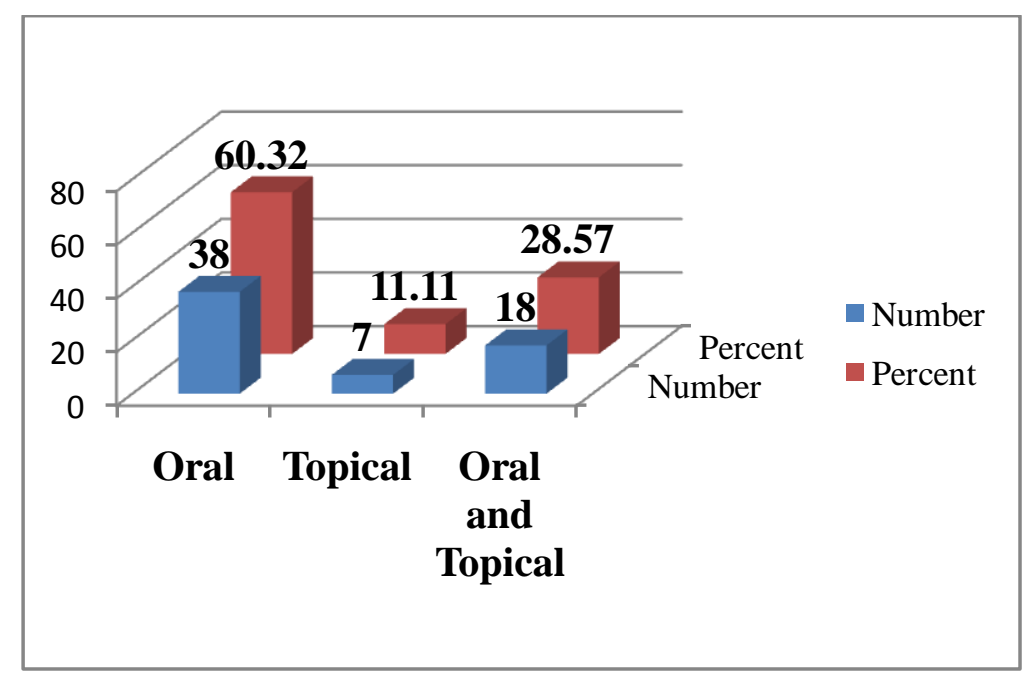

Figure 2:- Percent of routes of administration of different medicinal plant formulations.

\section{Discussion:-}

Plants have played a significant role in maintaining human health and in the treatment of different diseases. In our study, we recorded many medicinal plants used by the folk medicinal practitioners many of which have widely established medicinal value reported in many scientific journals and interestingly we found similar pattern of usage of medicinal plants in our study area.

Kavirajes of Sujanagar upazila of Pabna district frequently use Justicia adhatoda L.(Basok) in the treatment of cough and Calotropis gigantean (Akonda), Eclipta prostrate (Kalokeshi) in the treatment of asthma. It is reported in a review that the whole plant of Justicia adhatoda L is an ingredient of different cough formulation used in combination with Ginger (Zingiber officinale) and Tulsi (Ocimum sanctum) where it exerts its action as an expectorant and antispasmodic. The fluid extract and tincture were used in European medicine practitioners as an antispasmodic, expectorant and febrifuge (Dhankhar et al., 2011). Another study also reported broad spectrum antimicrobial activity of methanolic leaf extract of Justicia adhatoda L (Pa and Mathew, 2012). 
Many review articles published authentic information regarding the medicinal properties of different parts of Calotropis gigantean. Various phytoconstituents like Calotropin, Calotoxin, Choline, D-arabinose, D-glucosamine, B-Sitosterol are isolated from different parts of this plant which may be responsible for its different pharmacological activity. It is reported that different parts of this plant have antimicrobial, anti-cancer, anti-pyretic/analgesic and anti-asthmatic potential (Quazi et al, 2013, Sharma et al, 2012, Parvin et al, 2014). Folk medicine practitioners of Sujanagar upazila of Pabna district use various plant species in the treatment of gastrointestinal disorder. In our study, we found indigestion is treated with Leucas aspera( Dondokolosh ), Terminalia chebula Retz.(Horitoki), Aegle marmelos ( Bel), Carica papaya L.( Pepe). Such activities of these plants are recorded in many ethnomedicinal system and folk medicine practitioners of Tripura of India also uses Aegle marmelos in the treatment of indigestion, dysentery, constipation (Das et al., 2012). We also found the use of Carica papaya L both in the treatment of indigestion and constipation by the folk medicine practitioners.

There are many literatures published about the main phytoconstituents curcumin isolated from Curcuma longa $\mathrm{L}$ which mainly responsible for its different medicinal property like anti-inflammatory and anticancer activity (Zari and Zari, 2015, Araujo and Leon, 2001). In this study we found that kavirajes frequently use this plant mainly in the treatment of eczema and scabies.

Rural people suffering from STD frequently visit to folk medicine practitioners for their treatment and in our study we found that kavirajes of our study area use Ipomoea mauritiana Jacq.(Bhuikumra), Aloe barbadensis Miller(Ghritokumari), Heliotropium indicum (Hatisur) in the treatment of STD. Researchers found antimicrobial activity of different crude extracts of these plant that may be responsible for this therapeutic potential and they becomes a promising source of new antimicrobial agents (Devi et al., 2012, Osungunna and Adedeji, 2011). Abroma augusta have many therapeutic potential like root and root barks are used as uterine tonic, used in the treatment of amenorrhea, dysmenorrhea, extracts of different parts of plant are reported to have anti diabetic, anti-inflammatory, wound healing, hypolipidemic, antifungal, antibacterial and insecticidal activity (Gupta et al., 2011). In our study, we found the use of this plant in the treatment of infertility. Kavirajes also use Asparagus racemosus (satamuli) in the treatment of impotency of men.

Kavirajes provide wide range to treatment option as primary health care practitioners and rural people frequently visit to them for their many health ailments. In our study we found the use of many medicinal plants dispensed to different health disorder and formulated in different way by the kavirajes. Medicinal properties of these plants are well established and we found the use of same medicinal plants in different health disorders. Plants contain wide range of phytoconstituents and such phytoconstituents are mainly responsible for their various medicinal properties.

\section{Conclusion:-}

Knowledge about the medicinal properties of plants is usually transferred from generation to generation through informal educational system. Folk medicine practitioners are still playing a vital role in providing necessary remedy in this modern world and use of such plants in different health ailments are well established today. In our current study we recorded many plant species from different family which are also used by folk medicine practitioners of other parts of this country or even in other countries as well. Such documentation is very much necessary for preserving the knowledge and treatment pattern of different health ailments by folk medicine practitioners and such ethnomedicinal knowledge may lead to discovery of new drug compounds.

\section{References:-}

1. Araujo, C., Leon, L. 2001. Biological Activities of Curcuma longa L. Mem Inst Oswaldo Cruz., 96 (5): 723 728.

2. Das, S., Choudhury, MD. 2012. Ethnomedicinal uses of some traditional medicinal plants found in Tripura, India. J. Med. Plants Res., 6(35): 4908-4914.

3. Devi, DL., Srinivas, B., Rao, N. 2012. An evaluation antimicrobial activity of Aloe barbadensis Miller (Aloe vera) gel extract. J Pharm Biomed Sci., 21 (03): 1-4.

4. Dey, AK., Rashid, MM., Millat, MS., Rashid, MM. 2014. Ethnobotanical survey of medicinal plants used by traditional health practitioners and indigenous people in different districts of Chittagong division, Bangladesh. Int J Pharm Sci Invent., 3(7): 01-07. 
5. Dhankhar, S., Kaur, R., Ruhil, S., Balhara, M., Dhankhar, S., Chhilla, AK. 2011. A review on Justicia adhatoda: A potential source of natural medicine. Afr. J. Plant Sci., 5(11): 620-627.

6. District Statistics: Pabna District, 2011. Statistics and Informatics Division, Bangladesh Bureau of Statistics. http://www.bbs.gov.bd/WebTestApplication/userfiles/Image/PopCen2011/COMMUNITY_Pabna.pdf

7. Gupta, B., Nayak, S., Solanki, S. 2011. Abroma augusta Linn f: A review. Der Pharmacia Sinica., 2 (4):253261.

8. Joy, PP., Thomas, J., Mathew, S., Skaria, BP. 1998. Medicinal plants. Aromatic and Medicinal Plants Research Station, Kerala Agricultural University.

9. Osungunna, MO., Adedeji, KA. 2011. Phytochemical and antimicrobial screening of methanol extract of Heliotropium indicum leaf. J. Microbiol. Antimicrob., 3(8): 213-216.

10. Pa, R., Mathew, L. 2012. Antimicrobial activity of leaf extracts of Justicia adhatoda L. in comparison with vasicine. Asian Pac J Trop Biomed., S1556-S1560.

11. Parvin, S., Kader, MA., Chouduri, AU., Rafshanjani, MS., Haque, ME. 2014. Antibacterial, antifungal and insecticidal activities of the n-hexane and ethyl-acetate fractions of methanolic extract of the leaves of Calotropis gigantean Linn. J Pharmacogn Phytochem., 2 (5): 47-51.

12. Quazi, S., Mathur, K., Arora, S. 2013. Calotropisprocera: An overview of its phytochemistry and Pharmacology. IJOD., 1(2): 63-69.

13. Rahmatullah, M., Ferdausi, D., Mollik, MAH., Jahan, R., Chowdhury, MH., Haque, WH. 2010. A survey of medicinal plants used by kavirajes of Chalna area, Khulna district, Bangladesh. Afr J Trad CAM., 7 (2): 91 97.

14. Sahu, PK., Masih, V., Gupta, S., Sen, DL., Tiwari, A. 2014. Ethnomedicinal plants used in the healthcare systems of tribes of Dantewada, Chhattisgarh, India. Am J Plant Sci., 5: 1632-1643.

15. Salahuddin, M., Rakib, HH., Biplob, A., Islam, MS., Foyasal, MK., Tanvir, RZ. et al. 2015. Medicinal plants used by a traditional ayurvedic practitioner at Asadnagar village in Narsingdi District, Bangladesh. J Pharmacogn Phytochem., 4(2): 269-275.

16. Sharma, R., Thakur, GS., Sanodiya, BS., Savita, A., Pandey, M., Sharma, A., Bisen, PS. 2012. Therapeutic potential of Calotropis procera: A giant milkweed. IOSR J Pharm Biol Sci., 4(2): 42-57.

17. Tumpa, SI., Hossain, MI., Ishika, T. 2014. Ethnomedicinal uses of herbs by indigenous medicine practitioners of Jhenaidah district, Bangladesh. J Pharmacogn Phytochem., 3 (2):23-33.

18. Zari, ST., Zari, TA. 2015. A review of four common medicinal plants used to treat eczema. J Med Plants Res., 9(24): 702-711. 